Groups Geom. Dyn. 7 (2013), 557-576

DOI $10.4171 / \mathrm{GGD} / 197$
Groups, Geometry, and Dynamics

(C) European Mathematical Society

\title{
The dynamics of $\operatorname{Aut}\left(F_{n}\right)$ on redundant representations
}

\author{
Tsachik Gelander and Yair Minsky*
}

\begin{abstract}
We study some dynamical properties of the canonical $\operatorname{Aut}\left(F_{n}\right)$-action on the space $\mathcal{R}_{n}(G)$ of redundant representations of the free group $F_{n}$ in $G$, where $G$ is the group of rational points of a simple algebraic group over a local field. We show that this action is always minimal and ergodic, confirming a conjecture of A. Lubotzky. On the other hand for the classical cases where $G=\mathrm{SL}_{2}(\mathbb{R})$ or $\mathrm{SL}_{2}(\mathbb{C})$ we show that the action is not weak mixing, in the sense that the diagonal action on $\mathcal{R}_{n}(G)^{2}$ is not ergodic.
\end{abstract}

Mathematics Subject Classification (2010). 20G99, 22D40, 22F50, 37A25, 57M50.

Keywords. Aut $\left(F_{n}\right)$, character varieties, algebraic groups, 3-dimensional topology, dynamical decomposition, redundant representation, ergodicity.

\section{A short introduction}

Let $G$ be a group and consider $\operatorname{Hom}\left(F_{n}, G\right)$ - the representation space of the free group $F_{n}$ in $G$. The automorphism group $\operatorname{Aut}\left(F_{n}\right)$ acts naturally on $\operatorname{Hom}\left(F_{n}, G\right)$ by precompositions, inducing a canonical action of $\operatorname{Out}\left(F_{n}\right)$ on $\chi_{n}(G)=\operatorname{Hom}\left(F_{n}, G\right) / G$ - the space of conjugacy classes of representations. (The difference between this and the character variety $\operatorname{Hom}\left(F_{n}, G\right) / / G$ will not be important to us in this paper, and in particular they agree on the set of irreducible representations.) When $G$ has an additional structure (e.g. $G$ is algebraic, topological, measurable or finite, etc.) $\operatorname{Hom}\left(F_{n}, G\right)$ often inherits the structure from $G$ and the action respects the structure. For instance, if $G$ is a topological group $\operatorname{Hom}\left(F_{n}, G\right)$ is a topological space and $\operatorname{Aut}\left(F_{n}\right)$ acts by homeomorphisms. Similarly, if $G$ is a locally compact group, the Haar measure induces a measure on $\operatorname{Hom}\left(F_{n}, G\right)$ and $\operatorname{Aut}\left(F_{n}\right)$ preserves its measure class. Moreover, if $G$ is unimodular, $\operatorname{Aut}\left(F_{n}\right)$ preserves the measure. There are various reasons why people are interested in understanding the invariant subsets, and more generally the dynamics, of this action (we refer to Lubotzky's survey [Lu] for some of the motivations).

\footnotetext{
*Tsachik Gelander acknowledges the financial support from the European Research Council (ERC)/ grant agreement 203418, and the Israeli Science Foundation grant 1345/07. Yair Minsky acknowledges support from the National Science Foundation.
} 
W. M. Goldman conjectured that for every compact connected Lie group $G$, if $n \geq 3$, the $\operatorname{Aut}\left(F_{n}\right)$ action on $\operatorname{Hom}\left(F_{n}, G\right)$ is ergodic. As he pointed out for $n=2$ the action is not ergodic in general since the function $f \mapsto \operatorname{trace}\left(f\left(x y x^{-1} y^{-1}\right)\right)$, where $x, y \in F_{2}$ are free generators, is $\operatorname{Aut}\left(F_{2}\right)$-invariant and nonconstant if $G$ is noncommutative. In [Go], Goldman proved his conjecture for the case that all the simple factors of $G$ are locally isomorphic to SU(2). The general case of Goldman's conjecture was proved later in [Ge].

The compact-connected case is a bit misleading. For general $G$ one first restricts the attention to the subspace of epimorphisms, where in the context of topological groups, by epimorphism we mean a homomorphism with dense image:

$$
\operatorname{Epi}\left(F_{n}, G\right)=\left\{f \in \operatorname{Hom}\left(F_{n}, G\right): \overline{f\left(F_{n}\right)}=G\right\} .
$$

This is a measurable invariant subset, often even open, but in general its complement is also big and can be divided to subsets of the form $\operatorname{Epi}\left(F_{n}, H\right)$ for closed subgroups $H \leq G$. In the special case when $G$ is connected and compact almost every homomorphism has dense image (see [Ge], Lemma 1.10) and $\operatorname{Hom}\left(F_{n}, G\right)=\operatorname{Epi}\left(F_{n}, G\right)$ as measure spaces.

The first noncompact cases that were studied are $G=\mathrm{SL}_{2}(k)$ where $k$ is a local field. The mutual outcomes were somewhat surprising. More or less simultaneously, Y. Glasner [Gl] showed that when $k$ is nonarchimedean $\operatorname{Aut}\left(F_{n \geq 3}\right)$ acts ergodically on $\operatorname{Epi}\left(F_{n}, G\right)^{1}$, while Y. Minsky [Mi] showed that for $k=\mathbb{R}, \mathbb{C}$ the action is not ergodic. Minsky [Mi] defined the notion of primitive-stable homomorphism, and proved that the set of primitive-stable representations is open, containing the Schottky representations as well as a part of $\operatorname{Epi}\left(F_{n}, G\right)$, and the $\operatorname{action} \operatorname{of} \operatorname{Out}\left(F_{n}\right)$ is properly discontinuous on the set $\mathcal{P} S\left(F_{n}, G\right)$ of conjugacy classes of primitive-stable representations. On the other hand, in the nonarchimedean case, as one can deduce from Weidmann's theorem [We], [Gl], there are no primitive stable representations of $F_{n}$ in $\mathrm{SL}_{2}(k)^{2}$.

In an attempt to understand the global picture, and partly motivated by analogous results from finite group theory, A. Lubotzky [Lu] formulated the correct conjecture, namely that the action on the big subset of redundant representations is always ergodic. Recall:

Definition 1.1. A representation $\rho: F_{n} \rightarrow G$ is redundant if there exists a proper free factor $A$ of $F_{n}$ with $\rho(A)$ dense in $G$. We denote by $\mathcal{R}_{n}(G) \subset \operatorname{Hom}\left(F_{n}, G\right)$ the set of redundant representations.

When $G$ is a simple Lie group over a local field, the set $\mathcal{R}_{n}(G)$ is open (see Corollary 3.4).

\footnotetext{
${ }^{1}$ Assuming $\operatorname{char}(k) \neq 2$.

${ }^{2}$ Kapovich and Weidmann $[\mathrm{KW}]$ established a kind of generalization of Weidmann's theorem which applies in particular for $\mathrm{SL}_{2}(\mathbb{R}, \mathbb{C})$. It might be interesting to investigate the interplay between Minsky's and Kapovich-Weidmann's results.
} 
At first glance, Lubotzky's conjecture may seem wrong for the following reason. Note that a representation $\rho$ is redundant iff there is a free generating set $\left\{x_{1}, \ldots, x_{n}\right\}$ for $F_{n}$ such that $\rho\left(\left\langle x_{1}, \ldots, x_{n-1}\right\rangle\right)$ is dense in $G$. Call a representation $\rho: F_{n} \rightarrow G$ very redundant if for any free generating set $\left\{x_{1}, \ldots, x_{n}\right\}$ for $F_{n}$ and every $1 \leq i \leq n$, $\rho\left(\left\langle x_{j}: j \neq i\right\rangle\right)$ is dense in $G$ and let $\mathcal{V} \mathcal{R}_{n}(G)$ be the set of very redundant representations. Clearly $\mathcal{V} \mathcal{R}_{n}(G)$ is measurable, $\operatorname{Aut}\left(F_{n}\right)$-invariant and strictly contained in $\mathcal{R}_{n}(G)$. Hence if both $\mathcal{V} \mathcal{R}_{n}(G)$ and $\mathcal{R}_{n}(G) \backslash \mathcal{V} \mathcal{R}_{n}(G)$ have positive measure, the conjecture is false. However, while when $G$ is compact and $n \geq 3$, almost every representation is very redundant, when $G$ is noncompact one can show that there are no very redundant representations at all. Moreover, Lubotzky's conjecture is indeed true (see Theorem 2.1 below).

\section{Statement of the main results}

The following theorem confirms Lubotzky's conjecture:

Theorem 2.1. Let $k$ be a local field, $\mathbb{G}$ a Zariski connected simple $k$ group, and $G=\mathbb{G}(k)$ the group of $k$ points. If char $(k)>0$ assume further that the adjoint representation of $G$ is irreducible. Then the action of $\operatorname{Aut}\left(F_{n}\right)$ on $\mathcal{R}_{n}(G)$ is ergodic with respect to the Haar measure induced from $\operatorname{Hom}\left(F_{n}, G\right) \cong G^{n}$.

In case $G$ is compact and connected almost every representation of $F_{n \geq 3}$ is redundant, hence Theorem 2.1 recovers Theorem 1.6 of [Ge], namely that the action of $\operatorname{Aut}\left(F_{n}\right)$ on the representation variety $\operatorname{Hom}\left(F_{n}, G\right)$ is ergodic. Similarly, when $k$ is nonarchimedean and $G=\mathrm{SL}_{2}(k)$, almost every representation of $F_{n \geq 3}$ with dense image is redundant, as Glasner showed using Weidmann's theorem (see [Gl] for details). Hence the main result of $[\mathrm{Gl}]^{3}$ is also a special case of Theorem 2.1.

When $G$ is compact and $n \geq 3$, the action $\operatorname{Aut}\left(F_{n}\right) \propto \mathcal{R}_{n}(G)$ is even weakly mixing. This is because $\mathcal{R}_{n}(G)=\operatorname{Hom}\left(F_{n}, G\right)$ (up to measure 0 ) and $\operatorname{Hom}\left(F_{n}, G\right) \times$ $\operatorname{Hom}\left(F_{n}, G\right)$ is canonically identified with $\operatorname{Hom}\left(F_{n}, G \times G\right)$ while $G \times G$ is again a compact connected group (see [Ge] for more details). Somehow, perhaps unexpectedly, this stronger property of compact groups does not hold in general:

Theorem 2.2. Let $G=\mathrm{SL}_{2}(\mathbb{R})$ or $\mathrm{SL}_{2}(\mathbb{C})$, and $n \geq 3$. Then the action of $\operatorname{Out}\left(F_{n}\right)$ on $\overline{\mathcal{R}}_{n}(G)$ is not weakly mixing, in the sense that the diagonal action of $\operatorname{Out}\left(F_{n}\right)$ on $\overline{\mathcal{R}}_{n}(G) \times \overline{\mathcal{R}}_{n}(G)$ is not ergodic.

(Here $\overline{\mathcal{R}}_{n}(G)$ is the image of $\mathcal{R}_{n}(G)$ in $\chi_{n}(G)$. Note that nonergodicity in the quotient implies it for the action of $\operatorname{Aut}\left(F_{n}\right)$ upstairs).

\footnotetext{
${ }^{3}$ In [Gl] also the case of $G=\operatorname{Aut}(T)$, where $T$ is a regular tree, was treated. This case is not covered by 2.1 .
} 
Remark 2.3. We state and prove Theorem 2.2 for $^{\mathrm{SL}_{2}}$ since we will use 3-dimensional hyperbolic geometry in the proof. However the result extends immediately to every rank one simple Lie group $G$ (see also Remark 6.5).

Recall that an action on a topological space is minimal if every orbit is dense. The representation space $\operatorname{Hom}\left(F_{n}, G\right)$, hence also its subspace $\mathcal{R}_{n}(G)$, inherits a canonical topology from $G$. Moreover, the set $\mathcal{R}_{n}(G)$ is open (cf. Corollary 3.4 below). The following result is new even in the context of compact Lie groups (although a hint for it for compact $G$ is given in Remark 1.5 (1) of [Ge]).

Theorem 2.4. Let $G$ be as in Theorem 2.1. The action of $\operatorname{Aut}\left(F_{n}\right)$ on $\mathcal{R}_{n}(G)$ is minimal.

Remark 2.5. Theorems 2.1 and 2.4 remain true, and the proofs requires only minor changes, when $G$ is a general connected semisimple Lie group (not necessarily simple or linear algebraic). However, when $k$ is nonarchimedean and $G$ has more than one factor (i.e. semisimple but not simple) some parts of our arguments cannot be applied directly.

In analogy, when $G$ is a finite simple group, a classical result of Gilman [Gi] (for $n \geq 4$ ) and Evans [E] (for $n \geq 3$ ) states that $\operatorname{Aut}\left(F_{n}\right)$ acts transitively on $\mathcal{R}_{n}(G)$. As a consequence, the well known Weigold conjecture that $\operatorname{Aut}\left(F_{n}\right)$ acts transitively on $\operatorname{Epi}\left(F_{n}, G\right)$, or equivalently, that the associated product replacement graph is connected, reduces to the conjecture that every epimorphism is redundant, i.e. that $\operatorname{Epi}\left(F_{n}, G\right)=\mathcal{R}_{n}(G)$. Theorems 2.1 and 2.4 can be thought of as locally compact analogs of the Gilman-Evans theorem where instead of groups such as $\mathrm{SL}_{n}\left(\mathbb{F}_{q}\right)$ we consider $\mathrm{SL}_{n}(\mathbb{R})$ and $\mathrm{SL}_{n}\left(\mathbb{Q}_{p}\right)$.

\section{Remarks about dense subgroups}

In this section we form some basic results about dense subgroups that are relevant in the proofs of the main results.

Let $k$ be a local field (i.e. $\mathbb{R}, \mathbb{C}$, a finite extension of $\mathbb{Q}_{p}$ for some rational prime $p$, or the field $\mathbb{F}_{q}((t))$ of Laurent series over a finite field), $\mathbb{G}$ a Zariski connected simple algebraic group defined over $k$ and $G=\mathbb{G}(k)$ the group of $k$ rational points. In case $k$ is archimedean (i.e. $\mathbb{R}$ or $\mathbb{C}$ ), $G$ is a connected real analytic Lie group, and in case $k$ is a finite extension of $\mathbb{Q}_{p}, G$ is a $p$-adic analytic group. We denote by $g$ the Lie algebra of $G$, and by $\mathcal{A}$ the simple associative algebra spanned by the image of the adjoint representation Ad: $G \rightarrow \operatorname{Aut}(\mathrm{g})$. In the positive characteristic case, it is not always true that the representation Ad is irreducible, but we will restrict ourselves to that case, thus by Burnside's theorem $\mathcal{A}=\operatorname{End}(\mathfrak{g})$.

Let us first formulate some simple useful criterions for a subgroup of $G$ to be dense: 
An archimedean density criterion $(\boldsymbol{k}=\mathbb{R}$ or $\mathbb{C})$ : A subgroup $\Gamma \leq G$ is dense iff it is nondiscrete and $\operatorname{Ad}(\Gamma)$ generates $\mathcal{A}$.

The implication $\Rightarrow$ is obvious. For the other direction, denote $H=\bar{\Gamma}$ and $\mathfrak{h}=\operatorname{Lie}(H)$ its Lie algebra. One sees that $\mathfrak{h}$ is an ideal of $\mathfrak{g}$ (being $\operatorname{Ad}(\Gamma)$ invariant) of positive dimension (since $H$ is nondiscrete). As $\mathfrak{g}$ is simple, it follows that $\mathfrak{h}=\mathfrak{g}$ and hence $H=G$.

A nonarchimedean density criterion ( $\boldsymbol{k}$ is totally disconnected): A subgroup $\Gamma \leq$ $G$ is dense iff it is nondiscrete, unbounded, $\operatorname{Ad}(\Gamma)$ generates $\mathcal{A}$, and the entries of $\Gamma$ are not contained in a proper closed subfield of $k$.

To explain the nontrivial implication $\Leftarrow$, let us again denote $H=\bar{\Gamma}$ and $\mathfrak{h}=$ $\operatorname{Lie}(H)$. As in the archimedean case, $\mathfrak{h}$ is the full Lie algebra of $G$. It follows that $\operatorname{dim} H=\operatorname{dim} G$ and hence $H$ is Zariski dense. We claim furthermore that $H$ is open. This is a consequence of the following criterion of R. Pink [Pi]:

Lemma 3.1 (Pink's criterion). A compact subgroup of $G$ is open iff it is Zariski dense and not contained in $\mathbb{G}\left(k^{\prime}\right)$ for a proper closed subfield $k^{\prime}$.

Let $U$ be an open compact subgroup of $G$ and consider the compact group $H \cap U$. It is well known that $H \cap U$ is Zariski dense in $H$ and $\operatorname{Lie}(H \cap U)=\operatorname{Lie}(H)$ (see [PR], Lemma 3.2). Moreover since $H \nless \mathbb{G}\left(k^{\prime}\right)$ for every closed subfield $k^{\prime}<k$ while the adjoint representation is defined over the prime field, we deduce that $\operatorname{Ad}(H)$ is not contained in $\operatorname{Ad}(\mathbb{G})\left(k^{\prime}\right)$, and since, for $h \in H, \operatorname{Ad}(h)$ is determined by the restriction of $i_{h}: g \mapsto h g h^{-1}$ to the open (Zariski dense) set $H \cap U \cap h^{-1} U h$, we deduce that $H \cap U \not \mathbb{G}\left(k^{\prime}\right)$ (see [Gl] or [Sh] for more details). We deduce from Pink's criterion that $H$ is open.

Finally, the density criterion follows from the following result of Tits [Pr]:

Lemma 3.2. If $H \leq G$ is open and unbounded then $H=G$.

For the reader's convenience we include a proof of Lemma 3.2 (we believe this proof appears somewhere in the literature, but we are not aware of the correct source). Consider the unitary representation of $G$ on the separable Hilbert space $l_{2}(G / H)$ (or $l_{2}(G / H)^{0}$ if $\left.|G / H|<\infty\right)$ arising from the left action of $G$ on $G / H$. Clearly, there are no nonzero invariant vectors. However, if $[G: H]>1$, the unbounded subgroup $H$ admits a nontrivial invariant unit vector, in contrast to the Howe-Moore theorem. Hence $G=H$.

Here is another basic result:

Proposition 3.3. The set $\operatorname{Epi}\left(F_{n}, G\right)=\left\{f \in \operatorname{Hom}\left(F_{n}, G\right): f\left(F_{n}\right)\right.$ is dense in $\left.G\right\}$ is open in $\operatorname{Hom}\left(F_{n}, G\right)$, and nonempty provided $n \geq 2$. 
Proof. This well known when $k$ is archimedean (see [Ku1], [GZ], [BG]).

Suppose that $k$ is nonarchimedean, and let $f: F_{n} \rightarrow G$ be a homomorphism with dense image. Since the set of nonelliptic elements in $G$ is open, $f\left(F_{n}\right)$, as well as $f^{\prime}\left(F_{n}\right)$ for any $f^{\prime} \in \operatorname{Hom}\left(F_{n}, G\right)$ sufficiently close to $f$, contains a nonelliptic element and is hence unbounded. Moreover, $G$ admits an open finitely generated pro- $p$ group $K$ (see [BL]). It follows that the Frattini subgroup $F$ of $K$ is open. A subgroup of $K$ is dense in $K$ iff it intersects each of the finitely many open cosets of $F$ in $K$. This is clearly an open condition. This shows that $\overline{f^{\prime}\left(F_{n}\right)}$ for any $f^{\prime}$ sufficiently close to $f$ is open and unbounded. By Lemma 3.2, any such $f^{\prime}$ has dense image. Hence $\operatorname{Epi}\left(F_{n}, G\right)$ is open.

To show the second statement, we have to produce a 2-generated dense subgroup of $G$. First note that since the associative algebra $\mathcal{A}$ is finite dimensional, the set

$$
\{(a, b): \operatorname{Ad}(a), \operatorname{Ad}(b) \text { generates } \mathcal{A}\}
$$

is Zariski open in $G^{2}$, and since $G$ admits a 2-generated open subgroup (see [BL]) it is nonempty. Pick $(a, b)$ in this set such that $a$ is elliptic of infinite order and $b$ is nonelliptic. The closed field $k^{\prime}$ generated by the entries of $a$ is a local subfield of $k$. There are only finitely many intermediate fields between $k^{\prime}$ and $k$, hence, slightly deforming $b$ if necessary, we may assume that its entries are not contained in any of these intermediate fields. By the nonarchimedean density criterion sited above, $\langle a, b\rangle$ is dense.

As an immediate corollary we have:

Corollary 3.4. The set $\mathcal{R}_{n}(G)$ of redundant representations of $F_{n}$ in $G$ is also open in $\operatorname{Hom}\left(F_{n}, G\right)$. Moreover $\mathcal{R}_{n}(G) \neq \emptyset$ provided $n \geq 3$.

For a finite collection of elements $g_{1}, \ldots, g_{k} \in G$ we define $\Omega\left(g_{1}, \ldots, g_{k}\right)$ to be the set of elements $g$ in $G$ that together with $g_{1}, \ldots, g_{k}$ generates a dense subgroup of $G$ :

$$
\Omega\left(g_{1}, \ldots, g_{k}\right):=\left\{g \in G:\left\langle g_{1}, \ldots, g_{k}, g\right\rangle \text { is dense in } G\right\} .
$$

Then $\Omega\left(g_{1}, \ldots, g_{k}\right)$ is an open (possibly empty) subset of $G$. We will sometimes abuse notation and write $\Omega(S)$ for $\Omega\left(g_{1}, \ldots, g_{k}\right)$ when $S$ is the set $\left.S=\left\{g_{1}, \ldots, g_{k}\right\}\right)$. We will need the data that (under certain conditions) sets of this form intersect each other. In the archimedean case this will follow from:

Lemma 3.5. Suppose $k$ is archimedean. If $\Omega\left(g_{1}, \ldots, g_{k}\right)$ is nonempty, then there is an identity neighborhood $U$ in $G$, and a proper algebraic subvariety $X \subset G$ such that $\Omega\left(g_{1}, \ldots, g_{k}\right)$ contains $U \backslash X$.

Proof. If $\Omega\left(g_{1}, \ldots, g_{k}\right)$ is nonempty, picking $g \in \Omega\left(g_{1}, \ldots, g_{k}\right)$ we may find finitely many words $W_{i}, i=1, \ldots, m$ of $k+1$ variables such that $\operatorname{Ad}\left(W_{i}\left(g_{1}, \ldots, g_{k}, g\right)\right)$, 
$i=1, \ldots, m$ spans $\mathcal{A}$ as a vector space. Define

$$
X=\left\{g: \operatorname{span}\left\{\operatorname{Ad}\left(W_{i}\left(g_{1}, \ldots, g_{k}, g\right)\right), i=1, \ldots, m\right\} \neq \mathcal{A}\right\} .
$$

Let $V$ be a relatively compact Zassenhaus identity neighborhood of $G$ (see Chapter 8 in [R]). Recall that for every finite subset $S \subset V$ which generates a discrete subgroup, the Lie algebra generated by $\{\log s: s \in S\}$ is nilpotent. Let $U$ be a sufficiently small identity neighborhood in $G$ such that for any $u \in U, v \in V$ and every word $\mathcal{W}$ in $m$ letters of length $\leq \operatorname{dim} G+1$ we have

$$
\begin{aligned}
& \mathcal{W}\left(W_{1}\left(g_{1}, \ldots, g_{k}, v\right), \ldots, W_{m}\left(g_{1}, \ldots, g_{k}, v\right)\right) \\
& \quad u \mathcal{W}\left(W_{1}\left(g_{1}, \ldots, g_{k}, v\right), \ldots, W_{m}\left(g_{1}, \ldots, g_{k}, v\right)\right)^{-1} \in V .
\end{aligned}
$$

Now if $g \in U \backslash X$ then $\left\{\operatorname{Ad}\left(W_{i}\left(g_{1}, \ldots, g_{k}, g\right)\right), i=1, \ldots, m\right\}$ generates $\mathcal{A}$. Thus, by the archimedean density criterion, in order to prove that $\left\langle g_{1}, \ldots, g_{k}, g\right\rangle$ is dense, it is enough to show that it is nondiscrete. Suppose in contrary that it is discrete. Then for every $j \leq \operatorname{dim} G+1$ the Lie algebra

$$
\mathfrak{n}_{j}=\left\langle\log \left(\mathcal{W}_{g} \mathcal{W}^{-1}\right): \mathcal{W} \text { is a word in } W_{i}\left(g_{1}, \ldots, g_{k}, g\right) \text { of length } \leq j\right\rangle
$$

is nilpotent. But then for some $j \leq \operatorname{dim} G$ we have $\mathfrak{n}_{j}=\mathfrak{n}_{j+1}$ which forces the nontrivial nilpotent Lie algebra $\mathfrak{n}_{j}$ to be an ideal, since $\operatorname{Ad}\left(W_{i}\left(g_{1}, \ldots, g_{k}, g\right)\right)$ generates $\mathcal{A}$. A contradiction to the simplicity of $\mathfrak{g}$.

In particular the collection of these sets have the finite intersection property:

Corollary 3.6. In the archimedean case, every finite collection of nonempty sets of the form $\Omega\left(g_{1}, \ldots, g_{k}\right)$ has a nonempty intersection.

In the nonarchimedean case we prove a somewhat weaker result:

Lemma 3.7. Let $S_{j}, j=1, \ldots, r$, be a finite family of finite sets. Assume that $\Omega\left(S_{j}\right)$ is nonempty for every $j \leq r$ and that the groups $\left\langle S_{j}\right\rangle$ are simultaneously all nondiscrete or unbounded. Then $\bigcap_{j \leq r} \Omega\left(S_{j}\right) \neq \varnothing$.

Proof. The fact that $\Omega\left(S_{j}\right) \neq \varnothing$ implies that for all $g$ outside some proper algebraic subvariety $X_{j}$ the elements $\operatorname{Ad}(s), s \in S_{j} \cup\{g\}$, generate the algebra $\mathcal{A}$. Let $k_{j} \leq k$ be the closed subfield of $k$ generated by the entries of the elements of $S_{j}$, and let $\left\{k_{j, i}\right\}_{i=1}^{n_{j}}$ be the finite collection of proper local subfields in $k$ containing $k_{j}$ (if $k_{j}=k$ this collection is empty). If all the $\left\langle S_{j}\right\rangle$ are nondiscrete (resp. unbounded) pick

$$
g \in G \backslash\left(\bigcup_{j \leq r} X_{j} \cup \bigcup_{j \leq r, i \leq n_{j}} \mathbb{G}\left(k_{j, i}\right)\right)
$$

nonelliptic (resp. elliptic of infinite order). Then each of the groups $\left\langle s: s \in S_{j} \cup\right.$ $\{g\}\rangle$ satisfies the four condition of the nonarchimedean density criterion, i.e. it is unbounded, nondiscrete, its image under Ad generate $\mathcal{A}$ and its entries generate $k$. 
We will also need:

Lemma 3.8. Suppose $S_{i}, i=1,2$, are finite sets such that for both $i, \Omega\left(S_{i}\right) \neq \varnothing$ and each $S_{i}$ contains a nontorsion element. Then $\Omega\left(S_{1}\right) \cap \Omega\left(S_{2}\right) \neq \emptyset$.

Proof. Let $s_{i} \in S_{i}$ be a nontorsion element. In view of the previous lemma, it suffices to consider the case where $s_{1}$ is elliptic and $s_{2}$ is not. Then one deduces that there is an open set $V_{2} \subset \Omega\left(S_{2}\right)$ of elliptic elements. Moreover by choosing $V_{2}$ to be inside a small neighborhood of the identity, we can guarantee that the set $s_{2} V_{2}$ consists of nonelliptic elements. Let $X_{1}$ be the proper algebraic subvariety

$$
X_{1}=\left\{g \in G: \operatorname{Ad}(g), \operatorname{Ad}(s), s \in S_{1} \text { do not generate } \mathcal{A}\right\},
$$

and let $k_{1}, \ldots, k_{n}$ be the proper local subfields containing the field generated by the entries of $S_{1}$. Then

$$
s_{2} V_{2} \backslash\left(X_{1} \cup \bigcup_{i=1}^{n} \mathbb{G}\left(k_{i}\right)\right) \subset \Omega\left(S_{1}\right) \cap \Omega\left(S_{2}\right) .
$$

Indeed, if $g \in s_{2} V_{2} \backslash\left(X_{1} \cup \bigcup_{i=1}^{n} \mathbb{G}\left(k_{i}\right)\right)$ then $g$ together with $S_{1}$ generates an unbounded (since $g$ is nonelliptic) nondiscrete (since $s_{1}$ is elliptic of infinite order) subgroup whose image under Ad generates $\mathcal{A}$, and is not contained in $\mathbb{G}\left(k^{\prime}\right)$ for a proper local subfield $k^{\prime}<k$, hence is dense. On the other hand, $g$ together with $S_{2}$ generates a subgroup which contain $S_{2} \cup\left\{s_{2}^{-1} g\right\}$ and is hence dense.

For a finite set $S=\left\{g_{1}, \ldots, g_{k}\right\}$ let us also define:

$$
\widetilde{\Omega}(S):=\widetilde{\Omega}\left(g_{1}, \ldots, g_{k}\right):=\bigcap_{i=1, \ldots, k} \Omega\left(S \backslash\left\{g_{i}\right\}\right) .
$$

We will say that an ordered set (or an $n$-tuple) $S=\left(g_{1}, \ldots, g_{n}\right) \subset G^{n}$ is redundant if the element $f \in \operatorname{Hom}\left(F_{n}, G\right)$ defined by $f\left(x_{i}\right)=g_{i}$, where $\left\{x_{1}, \ldots, x_{n}\right\}$ is an arbitrary base, is redundant (this is independent of the choice of the generators $x_{i}$ ). For $\sigma \in \operatorname{Aut}\left(F_{n}\right)$ we will denote by $\sigma \cdot S$ the ordered set $\left(f\left(\sigma^{-1} \cdot x_{1}\right), \ldots, f\left(\sigma^{-1} \cdot x_{n}\right)\right)$. We will make use of the following:

Lemma 3.9. Let $S$ be an ordered set of size $n$ in $G$. Suppose that either

- $S$ is redundant, or

- $\langle S\rangle$ is dense and $S$ contains two nontorsion elements which are simultaneously elliptic or nonelliptic.

Then there is $\sigma \in \operatorname{Aut}\left(F_{n}\right)$ such that $\widetilde{\Omega}(\sigma \cdot S) \neq \varnothing$.

Proof. When $k$ is archimedean the lemma follows directly from Corollary 3.6 with $\sigma=1$, even if we only assume that $\langle S\rangle$ is dense.

If $k$ is nonarchimedean and the second condition holds, $\widetilde{\Omega}(S) \neq \emptyset$ by Lemma 3.7. 
Assume therefore that $k$ is nonarchimedean and $S$ is redundant. Up to replacing $S$ by $\sigma \cdot S$ for a suitable $\sigma \in \operatorname{Aut}\left(F_{n}\right)$ we may assume that $S=\left(g_{1}, \ldots, g_{n}\right)$ and $\left\langle g_{1}, \ldots, g_{n-1}\right\rangle$ is dense in $G$. Then the open set $\Omega\left(g_{2}, \ldots, g_{n-1}\right)$ is nonempty as it contains $g_{1}$, and hence we may multiply $g_{n}$ by some element $g^{\prime}$ belonging to the dense subgroup $\left\langle g_{1}, \ldots, g_{n-1}\right\rangle$ and obtain a non-torsion element $g^{\prime} g_{n}$ belonging to $\Omega\left(g_{2}, \ldots, g_{n-1}\right)$. Then we can find an element $g^{\prime \prime}$ belonging to the dense subgroup $\left\langle g_{2}, \ldots, g^{\prime} g_{n}\right\rangle$ such that $g^{\prime \prime} g_{1}$ is non-torsion of the same type (elliptic or non-elliptic) as $g^{\prime} g_{n}$, and belongs to the nonempty open set $\Omega\left(g_{2}, \ldots, g_{n-1}\right)$. Note that the ordered set $S^{\prime}=\left(g^{\prime \prime} g_{1}, g_{2}, \ldots, g_{n-1}, g^{\prime} g_{n}\right)$ was obtained from $S$ by a sequence of Nielsen transformations and is hence of the form $\tau \cdot S$ for some $\tau \in \operatorname{Aut}\left(F_{n}\right)$. Moreover, any subset of cardinality $n-1$ of $S^{\prime}$ contains either the first or the last element (which are both nonelliptic). Hence by Lemma $3.7 \widetilde{\Omega}\left(S^{\prime}\right) \neq \emptyset$.

\section{Minimality}

In this section we prove Theorem 2.4.

Given an element $\phi \in \mathcal{R}_{n}(G)$ and an open set $U \subset \mathcal{R}_{n}(G)$ we will find $\alpha \in$ $\operatorname{Aut}\left(F_{n}\right)$ with $\alpha \cdot \phi \in U$. By the definition of $\mathcal{R}_{n}(G)$, for an appropriate free generating set $\left\{x_{1}, \ldots, x_{n}\right\}$ we have that $\left\langle\phi\left(x_{i}\right): i \leq n-1\right\rangle$ is dense in $G$. Moreover acting by Nielsen transformations which change only the last coordinate, and then by Nielsen transformations which change only the first coordinate, we may change $\phi$ so that in addition to the previous condition, $\phi\left(x_{n}\right) \in \Omega\left(\phi\left(x_{2}\right), \ldots, \phi\left(x_{n-1}\right)\right)$ and $\phi\left(x_{1}\right)$ is nontorsion. Moving $U$ by some appropriate element of $\operatorname{Aut}\left(F_{n}\right)$ we may furthermore assume that for some $\phi^{\prime} \in U,\left\langle\phi^{\prime}\left(x_{i}\right): i \leq n-1\right\rangle$ is dense, and $\phi^{\prime}\left(x_{1}\right)$ is nontorsion as well.

We will say that an element $\psi \in \mathcal{R}_{n}(G)$ links an element $\varphi \in \mathcal{R}_{n}(G)$ if for every $k<n$, the group

$$
\left\langle\varphi\left(x_{1}\right), \ldots, \varphi\left(x_{k-1}\right), \psi\left(x_{k+1}\right), \ldots, \psi\left(x_{n}\right)\right\rangle
$$

is dense in $G$. The set

$$
€(\varphi):=\left\{\psi \in \mathcal{R}_{n}(G): \psi \text { links } \varphi\right\}
$$

is always open.

We claim that $\mathrm{\complement}(\phi)$ is contained in the closure of the orbit $\operatorname{Aut}\left(F_{n}\right) \cdot \phi$ (and the analog statement for $\left.\phi^{\prime}\right)$. Indeed, given $\psi \in \mathrm{七}(\phi)$, since $\left\langle\phi\left(x_{i}\right): i<n\right\rangle$ is dense and $\psi\left(x_{n}\right)$ belongs, by definition, to $\Omega\left(\phi\left(x_{1}\right), \ldots, \phi\left(x_{n-2}\right)\right)$, for an appropriate composition of Nielsen transformations which act on the $n$-th coordinate by multiplying it with other coordinates, we obtain an element $\sigma_{n}$ for which $\sigma_{n} \cdot \phi\left(x_{i}\right)=\phi\left(x_{i}\right)$ for $i<n$ and $\sigma_{n} \cdot \phi\left(x_{n}\right)$ is arbitrarily close to $\psi\left(x_{n}\right)$ and belongs to $\Omega\left(\phi\left(x_{1}\right), \ldots, \phi\left(x_{n-2}\right)\right)$. After that, using the density of $\left\langle\sigma_{n} \cdot \phi\left(x_{1}\right), \ldots, \sigma_{n} \cdot \phi\left(x_{n-2}\right), \sigma_{n} \cdot \phi\left(x_{n}\right)\right\rangle$ we may 
find an element $\sigma_{n-1} \in \operatorname{Aut}\left(F_{n}\right)$ which is a composition of Nielsen transformations acting on the $(n-1)$-th coordinate by multiplying it by the others, such that $\sigma_{n-1} \sigma_{n} \cdot \phi\left(x_{i}\right)=\sigma_{n} \phi\left(x_{i}\right)$ for $i \neq n-1$, and $\sigma_{n-1} \sigma_{n} \cdot \phi\left(x_{n-1}\right)$ belongs to $\Omega\left(\phi\left(x_{1}\right), \ldots, \phi\left(x_{n-3}\right), \sigma_{n} \cdot \phi\left(x_{n}\right)\right)$ and is arbitrarily close to $\psi\left(x_{n-1}\right)$. Repeating this procedure recursively for the lower indices we obtain an element $\sigma_{1} \sigma_{2} \ldots \sigma_{n}$ which moves $\phi$ arbitrarily close to $\psi$.

Next observe that $€(\phi) \cap €\left(\phi^{\prime}\right) \neq \emptyset$. Indeed, by Lemma 3.8,

$$
\Omega\left(\phi\left(x_{1}\right), \ldots, \phi\left(x_{n-2}\right)\right) \cap \Omega\left(\phi^{\prime}\left(x_{1}\right), \ldots, \phi^{\prime}\left(x_{n-2}\right)\right) \neq \emptyset .
$$

Pick $g_{n}$ in this set. Again, by Lemma 3.8,

$$
\Omega\left(\phi\left(x_{1}\right), \ldots, \phi\left(x_{n-3}\right), g_{n}\right) \cap \Omega\left(\phi^{\prime}\left(x_{1}\right), \ldots, \phi^{\prime}\left(x_{n-3}\right), g_{n}\right) \neq \emptyset,
$$

so pick $g_{n-1}$ in this intersection. In a recursive way we define $g_{i}$ for the lower indices. Defining $\psi$ by $\psi\left(x_{i}\right)=g_{i}, i=1, \ldots, n$ we obtain an element $\psi$ which links both $\phi$ and $\phi^{\prime}$.

Since $€(\phi) \cap €\left(\phi^{\prime}\right)$ is open nonempty and contained in $\overline{\operatorname{Aut}\left(F_{n}\right) \cdot \phi^{\prime}}$, we may find $\sigma \in \operatorname{Aut}\left(F_{n}\right)$ such that $\sigma \cdot \phi^{\prime} \in \mathrm{Ł}(\phi) \cap €\left(\phi^{\prime}\right)$. Similarly we can find $\tau \in \operatorname{Aut}\left(F_{n}\right)$ such that $\tau \cdot \phi \in \mathrm{Ł}(\phi) \cap \mathrm{Ł}\left(\phi^{\prime}\right) \cap \sigma \cdot U$. It follows that $\sigma^{-1} \tau \cdot \phi \in U$.

\section{Ergodicity}

We are now in a position to prove Theorem 2.1. Let $\left\{x_{1}, \ldots, x_{n}\right\}$ be a generating set of $F_{n}$.

We will say that an $n$-tuple $\left(g_{1}, \ldots, g_{n}\right) \in G^{n}$ is strongly redundant if every $(n-1)$-subtuple generates a dense subgroup of $G$. We first claim that if $n \geq 3$ then there exists a strongly redundant $n$-tuple. To see this, start with an arbitrary $(n-1)$ subtuple $\left(g_{1}, \ldots, g_{n-1}\right)$ which generates a dense subgroup. If $k$ is archimedean, by Corollary 3.6, $\widetilde{\Omega}\left(g_{1}, \ldots, g_{n-1}\right) \neq \emptyset$ and the claim follows, using $\left(g_{1}, \ldots, g_{n-1}, g\right)$ for any $g \in \widetilde{\Omega}\left(g_{1}, \ldots, g_{n-1}\right)$. If $k$ is nonarchimedean, slightly deforming the $g_{i}, i \leq$ $n-1$ we may assume that they are all nontorsion. Then again $\widetilde{\Omega}\left(g_{1}, \ldots, g_{n-1}\right) \neq \emptyset$; for $n=3$ this follows from Lemma 3.8, while for $n>3$ from Lemma 3.9 since at lease two of the $(\geq 3)$ elements $\left(g_{1}, \ldots, g_{n-1}\right)$ are simultaneously elliptic or not.

The set $S \mathcal{R}$ of strongly redundant $n$-tuples is open in $G^{n}$. We will call a subset of $S \mathcal{R}$ of the form $\prod_{i=1}^{n} U_{i}$ a strongly redundant open cube. We shall identify $\operatorname{Hom}\left(F_{n}, G\right)$ with $G^{n}$ via the map $f \mapsto\left(f\left(x_{1}\right), \ldots, f\left(x_{n}\right)\right)$. In particular, we shall say that a representation $f \in \operatorname{Hom}\left(F_{n}, G\right)$ is strongly redundant if $\left(f\left(x_{1}\right), \ldots, f\left(x_{n}\right)\right)$ is a strongly redundant $n$-tuple.

Let $A \subset \mathcal{R}_{n}(G)$ be a measurable $\operatorname{Aut}\left(F_{n}\right)$ almost invariant subset. We wish to show that $A$ is either null or conull. Replacing $A$ by the countable intersection $\bigcap_{\sigma \in \operatorname{Aut}\left(F_{n}\right)} \sigma \cdot A$ we may assume that it is precisely invariant rather than almost invariant. 
Let us fix once and for all a strongly redundant open cube $U=\prod_{i=1}^{n} U_{i}$. Arguing as in the proof of [Ge], Theorem 1.6, one deduces that the intersection of $A$ with $U$ is either null or conull in $U$. Indeed, assuming the contrary, one derives from Fubini's theorem that for some index $i_{0} \in\{1, \ldots, n\}$ and a choice of $u_{j} \in U_{j}$ for every $j \neq i_{0}$, the set

$$
\left\{u \in U_{i_{0}}:\left(u_{1}, \ldots, u_{i_{0}-1}, u, u_{i_{0}+1}, \ldots, u_{n}\right) \in A\right\}
$$

is neither null nor conull in $U_{i_{0}}$ and hence the set

$$
Y=\left\{g \in G:\left(u_{1}, \ldots, u_{i_{0}-1}, g, u_{i_{0}+1}, \ldots, u_{n}\right) \in A\right\}
$$

is neither null nor conull in $G$. However, since $A$ is invariant under Nielsen transformations, $Y$ is invariant under the left action of the group $\left\langle u_{i}, i \neq i_{0}\right\rangle$. But this group is dense and hence acts ergodically on $G$, a contradiction. Thus, up to replacing $A$ by its complement, we may assume that $A \cap U$ is null.

Now let $f \in \mathcal{R}_{n}(G)$ be an arbitrary redundant representation. Since the action of $\operatorname{Aut}\left(F_{n}\right)$ on $\mathcal{R}_{n}(G)$ preserves the topology and is minimal, for some $\sigma \in \operatorname{Aut}\left(F_{n}\right)$ we have $\sigma \cdot f \in U$ and hence $\sigma^{-1} U$ is an open neighborhood of $f$ which meets $A$ in a null set.

Since $\mathcal{R}_{n}(G)$ is homeomorphic to an open subset of $G^{n}$ it is second countable, and thus can be covered by a countable union of open sets, each meets $A$ in a null set. It follows that $A$ is null.

\section{Nonmixing}

In this section we consider the case of $G=\operatorname{SL}(2, \mathbb{C})$ and $G=\operatorname{SL}(2, \mathbb{R})$, where hyperbolic geometry gives us additional structure. In these cases we show that the action on $\mathcal{R}_{n}(G)$, in spite of being minimal and ergodic, is not weakly mixing in a suitable sense. We will consider the action of $\operatorname{Out}\left(F_{n}\right)$ on the space of $\chi_{n}(G)=$ $\operatorname{Hom}\left(F_{n}, G\right) / G$, letting $\overline{\mathcal{R}}_{n}(G)=\mathcal{R}_{n}(G) / G$ be the space of conjugacy classes of redundant representations.

Theorem 6.1. The action of $\operatorname{Out}\left(F_{n}\right)$ on $\overline{\mathcal{R}}_{n}(G)$, for $n \geq 3$, is not weakly mixing. Indeed, the diagonal action is not ergodic on $\overline{\mathcal{R}}_{n}(G) \times \overline{\mathcal{R}}_{n}(G)$, and in fact there is an open nonempty invariant subset of $\overline{\mathcal{R}}_{n}(G) \times \overline{\mathcal{R}}_{n}(G)$ on which $\operatorname{Out}\left(F_{n}\right)$ acts properly discontinuously.

We begin by recalling some definitions.

If $X$ is a generating set for $F_{n}$ and $A$ a set of cyclically reduced words in $F_{n}$, the Whitehead graph $\mathrm{Wh}(A, X)$ is defined as follows: The vertex set of $\mathrm{Wh}(A, X)$ is set $X^{ \pm}=\left\{x, x^{-1}: x \in X\right\}$. An (unoriented) edge $[a b]$ appears whenever $a b^{-1}$ is a subword of a cyclic permutation of a word of $A$ (and in addition $\left[a a^{-1}\right]$ is an 
edge whenever $A$ contains the length 1 word $a$ ). See Whitehead [Wh1], [Wh2] and Stallings [St].

For a single word write $\mathrm{Wh}(\gamma, X)=\mathrm{Wh}(\{\gamma\}, X)$. If $\alpha$ is a collection of loops in the handlebody of genus $n$, or conjugacy classes in $F_{n}$, define $\mathrm{Wh}(\alpha, X)$ to be $\mathrm{Wh}(A, X)$ for (any) set of cyclically reduced words representing $\alpha$.

Note that $\mathrm{Wh}(A \cup B, X)=\mathrm{Wh}(A, X) \cup \mathrm{Wh}(B, X)$ where "union" of graphs means union followed by identification of duplicate edges.

An element of $F_{n}$ is primitive if it is a member of a free generating set. Whitehead gave the following property as part of an algorithm for deciding primitivity in $F_{n}$ :

Basic Lemma (Whitehead). If $\gamma$ is a primitive cyclically reduced element then, for any generating set $X, \mathrm{Wh}(\gamma, X)$ is either disconnected or has a cutpoint.

Primitive-stable pairs. Since $G$ acts on $\mathbb{H}^{3}$ in both the real and complex case, we can consider as in [Mi] the geometric properties of representations $\operatorname{in} \operatorname{Hom}\left(F_{n}, G\right)$.

We define a subset $\mathcal{P} S_{n}^{2} \subset \chi_{n}(G)^{2}$ as follows. Recall from [Mi] that for each $\rho \in \operatorname{Hom}\left(F_{n}, G\right)$ and basepoint $x \in \mathbb{H}^{3}$ there is an orbit map $\tau_{\rho, x}: F_{n} \rightarrow \mathbb{H}^{3}$, namely $g \mapsto \rho(g) x$. Fixing a set of generators we also extend $\tau_{\rho, x}$ to the corresponding Cayley graph of $F_{n}$, by mapping edges to geodesic segments.

Recall also that every nontrivial element of $F_{n}$ has an axis in the Cayley graph, and let $\mathcal{P}$ denote the set of axes of primitive elements.

Given a constant $K$ and basepoint $x$, let $\mathcal{A}(K, x, \rho)$ denote the set of axes which $\tau_{\rho, x}$ maps $K$-quasi-geodesically to $\mathbb{H}^{3}$. (A map $f: \mathbb{R} \rightarrow Y$ to a metric space $Y$ is $K$-quasi-geodesic if $|s-t| / K-K \leq d_{Y}(f(s), f(t)) \leq K|s-t|+K$.) In [Mi], $\mathcal{P} S_{n}$ was defined as the set of (conjugacy classes of) representations for which there exists $K, x$ such that $\mathcal{P} \subset \mathcal{A}(K, x, \rho)$.

Now define $\mathcal{P} S_{n}^{2}$ as the set of pairs $\left(\left[\rho_{1}\right],\left[\rho_{2}\right]\right)$ such that there exist representatives $\rho_{1}, \rho_{2}, K>0$, and $x \in \mathbb{H}^{3}$ with

$$
\mathcal{P} \subset \mathcal{A}\left(K, x, \rho_{1}\right) \cup \mathcal{A}\left(K, x, \rho_{2}\right)
$$

We state some basic properties of this set:

Lemma 6.2. Let $n \geq 3$ and $G=\operatorname{SL}(2, \mathbb{C})$ or $\operatorname{SL}(2, \mathbb{R})$.

(1) $\mathcal{P} S_{n}^{2}$ is open.

(2) $\mathcal{P} S_{n}^{2}$ is $\operatorname{Out}\left(F_{n}\right)$-invariant.

(3) The action on $\mathcal{P} S_{n}^{2}$ is properly discontinuous.

Proof. The proof proceeds essentially as in [Mi] for the corresponding facts for $\mathcal{P} S_{n}$. We give sketches.

(1) In [Mi] in the proof of Theorem 3.2, the following stability property is given: 
Lemma 6.3. Given $K, x$ and $\rho$ there exists $K^{\prime}$ and a neighborhood $U$ of $\rho$ such that

$$
\mathcal{A}(K, x, \rho) \subset \mathcal{A}\left(K^{\prime}, x, \rho^{\prime}\right)
$$

for all $\rho^{\prime} \in U$

The idea of this is the following: Let $A$ be an axis in $\mathcal{A}(K, x, \rho)$. Its $\tau_{\rho, x}$ image is composed of a biinfinite sequence of segments such that successive ones are related by (conjugates of) $\rho$-images of generators. The $K$-quasi geodesic property implies that this axis makes "definitely fast" progress in $\mathbb{H}^{3}$, which means the following: There exists $k$ depending only on $K, x$ and $\rho$ such that any pair of segments separated by $k$ steps are separated by a hyperplane in $\mathbb{H}^{3}$ such that the sequence of hyperplanes separate each other and are pairwise separated by a distance strictly greater than 0 . A small perturbation of $\rho$ affects each sequence of $k$ generators by a small amount, and hence preserves this hyperplane property (but changes the constants). Hence the $\tau_{\rho^{\prime}, x}$ image of $A$ is $K^{\prime}$-quasi-geodesic, where $K^{\prime}$ depends on $K, x$ and how close $\rho^{\prime}$ is to $\rho$.

With this lemma in hand, suppose $\left(\left[\rho_{1}\right],\left[\rho_{2}\right]\right) \in \mathcal{P} S_{n}^{2}$ and let $x, K$ be such that $\mathcal{P}$ is contained in $\mathcal{A}\left(K, x, \rho_{1}\right) \cup \mathcal{A}\left(K, x, \rho_{2}\right)$. Let $U_{1}, K_{1}$ and $U_{2}, K_{2}$ be given by Lemma 6.3 for $\rho_{1}$ and $\rho_{2}$ respectively, and let $K^{\prime}=\max \left(K_{1}, K_{2}\right)$. Then we have

$$
\mathcal{P} \subset \mathcal{A}\left(K^{\prime}, x, \rho_{1}^{\prime}\right) \cup \mathcal{A}\left(K^{\prime}, x, \rho_{2}^{\prime}\right)
$$

for all $\left(\rho_{1}^{\prime}, \rho_{2}^{\prime}\right) \in U_{1} \times U_{2}$. It follows, letting $U_{i}^{\prime}$ denote the image of $U_{i}$ in $\chi_{n}(G)$, that $U_{1}^{\prime} \times U_{2}^{\prime} \subset \mathcal{P} S_{n}^{2}$. (Note that $\operatorname{Hom}\left(F_{n}, G\right) \rightarrow \chi_{n}(G)$, being a quotient by a group action, is an open map, so that $U_{1}^{\prime}$ and $U_{2}^{\prime}$ are open.)

(2) Suppose $\left(\left[\rho_{1}\right],\left[\rho_{2}\right]\right) \in \mathcal{P S}_{n}^{2}$. Any $\psi \in \operatorname{Aut}\left(F_{n}\right)$ acts by quasi-isometry on the Cayley graph of $F_{n}$, and it follows that the image of the axis of any $g \in F_{n}$ is a quasi-geodesic (with constants depending on $\psi$ ) that shadows the axis of $\psi(g)$. Now if $g$ is primitive, so is $\psi(g)$, so that the axis of $\psi(g)$ is in $\mathcal{A}\left(K, x, \rho_{i},\right)$ for $i=1$ or 2. But this means, for $K^{\prime}$ depending on $K$ and the quasi-isometry constant of $\psi$, that the axis of $g$ is in $\mathcal{A}\left(K^{\prime}, x, \rho_{i} \circ \psi\right)$. Hence $\left(\left[\rho_{1} \circ \psi\right],\left[\rho_{2} \circ \psi\right]\right) \in \mathcal{P} S_{n}^{2}$ too.

(3) (Following the argument of Theorem 3.3 of [Mi].)

For a conjugacy class $c \in F_{n}$ let $\|c\|$ denote the length of a cyclically reduced representative, or equivalently the translation length of any representative of $c$ on its axis in the Cayley graph, and let $\ell_{\rho}(c)$ denote the translation length of the conjugacy class $\rho(c)$ in $\mathbb{H}^{3}$. If the axis of (any representative of) $c$ is in $\mathcal{A}(K, x, \rho)$ then $\ell_{\rho}(c) /\|c\|$ is bounded above and below by positive constants depending on $K, x$.

So now if $\left(\left[\rho_{1}\right],\left[\rho_{2}\right]\right) \in \mathcal{P} S_{n}^{2}$, all primitive conjugacy classes $c$ satisfy such a bound either on $\ell_{\rho_{1}}(c) /\|c\|$ or on $\ell_{\rho_{2}}(c) /\|c\|$. Moreover these bounds vary by a bounded ratio for a fixed $c$ and small perturbations of the representation, as a consequence of Lemma 6.3.

Now let $E$ be a compact subset in $\mathcal{P} S_{n}^{2}$. The above gives us uniform upper and lower bounds either on $\ell_{\rho_{1}}(c) /\|c\|$ or on $\ell_{\rho_{2}}(c) /\|c\|$, for each primitive $c$, over all 
of $E$. If $\Phi \in \operatorname{Out}\left(F_{n}\right)$ such that $\Phi(E) \cap E \neq \emptyset$, let $\left(\rho_{1}, \rho_{2}\right)$ be in this intersection. For each primitive $c$ we obtain a bound of the form $\ell_{\rho_{i}}(c) \leq b_{1}\|c\|$ for $i=1$ and $i=2$, simply because the maps $\tau_{\rho_{i}, x}$ have uniform Lipschitz bounds on $E$. Since $\left(\rho_{1}, \rho_{2}\right) \in \Phi(E)$ we also obtain a bound of the form $\|\Phi(c)\| \leq b_{2} \ell_{\rho_{i}}(c)$, for at least one $i \in\{1,2\}$. Putting these together we obtain a uniform upper bound on $\|\Phi(c)\| /\|c\|$. This suffices, e.g. by Lemma 3.4 of [Mi], to restrict $\Phi$ to a finite set in $\operatorname{Out}\left(F_{n}\right)$. It follows that the action is properly discontinuous.

Lemma 6.2 tells us that $\mathcal{P} S_{n}^{2} \cap \overline{\mathcal{R}}_{n}(G) \times \overline{\mathcal{R}}_{n}(G)$ is the set required by Theorem 6.1, provided we can prove that it is non-empty.

The proof of this will take a somewhat different form when $G=\operatorname{SL}(2, \mathbb{C})$ and $G=\operatorname{SL}(2, \mathbb{R})$. Although it suffices in fact to prove the real case since it embeds in the complex case, we give a separate proof in the complex case since the theory of hyperbolic 3-manifolds can be applied, giving a more flexible and geometric construction.

6.1. The complex case. The proof will hinge on the following construction:

Lemma 6.4. Let $H$ be the genus $n$ handlebody for $n \geq 3$. There exist simple loops $\alpha_{1}$ and $\alpha_{2}$ on $\partial H$, and a generating set $X$ for $\pi_{1}(H)$, such that a representative of each $\alpha_{i}$ in $F_{n}$ is contained in a proper free factor, but the Whitehead graph of the union, $\operatorname{Wh}\left(\left\{\alpha_{1}, \alpha_{2}\right\}, X\right)$, is connected and without cutpoints.

Moreover, each $\alpha_{i}$ can be chosen so that $H$ admits a geometrically finite hyperbolic structure for which $\alpha_{i}$ is the unique parabolic.

Proof. We can write $H$ as a boundary connected sum (i.e. gluing along disks)

$$
H=T_{1} \cup H^{\prime} \cup T_{2}
$$

where $H^{\prime}$ is a handlebody of genus $n-2$ and $T_{1}$ and $T_{2}$ are handlebodies of genus 1 , i.e. solid tori. We then rearrange $H$ as a union of overlapping handlebodies of genus $n-1, H_{1}=T_{1} \cup H^{\prime}$ and $H_{2}=T_{2} \cup H^{\prime}$.

Choose generators $X=\left\{X_{1}, \ldots, X_{n}\right\}$ for $F_{n}=\pi_{1}(H)$ so that $X_{1}$ generates $\pi_{1}\left(T_{1}\right), X_{2}$ generates $\pi_{1}\left(T_{2}\right)$, and the rest generate $\pi_{1}\left(H^{\prime}\right)$. Now for $i=1,2$ suppose that $\gamma_{i}$ is an element of $\pi_{1}\left(H_{i}\right)$ whose Whitehead graph $\mathrm{Wh}\left(\gamma_{i},\left\{X_{i}, X_{3}, \ldots, X_{n}\right\}\right)$ is connected and without cutpoints. Considered with respect to all generators, $\mathrm{Wh}\left(\gamma_{1}, X\right)$ is disconnected because it has no edges incident to $X_{2}^{ \pm}$, and indeed $\gamma_{1}$ is contained in the proper free factor $\left\langle X_{1}, X_{3}, \ldots, X_{n}\right\rangle$. The corresponding statements hold for $\gamma_{2}$.

However, $\operatorname{Wh}\left(\left\{\gamma_{1}, \gamma_{2}\right\}, X\right)$ is the union of two connected graphs without cutpoints, which together meet every vertex and intersect along at least two vertices (since there are two vertices per generator). It follows that $\mathrm{Wh}\left(\left\{\gamma_{1}, \gamma_{2}\right\}, X\right)$ is both connected and without cutpoints. 
Now as discussed in [Mi], we may select $\alpha_{i}$ in the Masur domain of $H_{i}$, and this will imply that the Whitehead graph of $\alpha_{i}$ with respect to some generating set will be connected without cutpoints. Applying a homeomorphism if necessary, we may assume that the generating set is the one we have already fixed. Moreover, being in the Masur domain implies that $H_{i}$ admits a geometrically finite hyperbolic structure for which $\alpha_{i}$ is the sole parabolic.

One can always choose representatives of such curves on $H_{1}$ or on $H_{2}$ which are disjoint from the gluing disks, and hence they can be made to lie on the boundary of $H$. Finally, the geometrically finite representations we have on $H_{i}$ can be extended to representations on $H$ which are still geometrically finite with the $\alpha_{i}$ as sole parabolics - this is an immediate consequence of the Klein Combination Theorem which gives conditions on constructing free products of Kleinian groups (in this case, the factors are $\rho_{i}\left(\pi_{1}\left(H_{i}\right)\right)$ and a hyperbolic cyclic group corresponding to $\left.\pi_{1}\left(T_{3-i}\right)\right)$ and describes the type of the resulting group. See Klein [Kl] and Maskit [Ma].

Let $\alpha_{1}, \alpha_{2}$ and the generating set $X$ be as in Lemma 6.4, and let $\rho_{1}, \rho_{2}: \pi_{1}(H) \rightarrow$ $G$ be representations corresponding to the geometrically finite structures the lemma provides for $\alpha_{1}$ and $\alpha_{2}$. We claim that $\left(\left[\rho_{1}\right],\left[\rho_{2}\right]\right) \in \mathcal{P} S_{n}^{2}$. The proof follows the argument in $[\mathrm{Mi}]$ with minor variations:

The property of geometric finiteness implies that each quotient manifold $N_{i}=$ $\mathbb{H}^{3} / \rho_{i}\left(F_{n}\right)$ contains a convex core $C_{i}$ which is not compact, but can be written as $H_{i} \cup Q_{i}$ where $H_{i}$ is a compact handlebody and $Q_{i}$ is a cusp neighborhood associated to $\alpha_{i}$. All closed geodesics in $N_{i}$ are contained in $C_{i}$, and if a closed geodesic $\gamma$ penetrates deeply into $Q_{i}$ then the corresponding reduced word in $F_{n}$ contains a high power of the reduced form of $\alpha_{i}$. This is shown in the proof of Theorem 4.1 of [Mi].

Hence, after possibly enlarging $H_{i}$, we have the following property: If a conjugacy class $\gamma$ in $F_{n}$ has geodesic representative $\gamma^{i}$ which is not contained in $H_{i}$, then $\mathrm{Wh}(\gamma, X)$ contains $\mathrm{Wh}\left(\alpha_{i}, X\right)$. On the other hand by compactness there exists $K$ and $x \in \mathbb{H}^{3}$ so that if $\gamma^{i}$ is contained in $H_{i}$ then the axis of $\gamma^{i}$ is mapped $K$ quasi-geodesically by $\tau_{\rho_{i}, x}$. Let $P_{i}$ be the set of conjugacy classes whose geodesic representatives in $N_{i}$ are contained in $H_{i}$, and hence satisfy the quasi-geodesic condition with respect to $\rho_{i}$.

What we have shown is that any element not in $P_{1} \cup P_{2}$ has Whitehead graph containing $\mathrm{Wh}\left(\left\{\alpha_{1}, \alpha_{2}\right\}, X\right)$. Since this graph is connected and without cutpoints, Whitehead's Lemma tells us that such an element cannot be primitive. We conclude that $P_{1} \cup P_{2}$ cover all the primitive elements, so that $\left(\left[\rho_{1}\right],\left[\rho_{2}\right]\right) \in \mathcal{P S}_{n}^{2}$.

Now, since $\alpha_{i}$ is contained in a proper free factor $B_{i}<F_{n},\left.\rho_{i}\right|_{B_{i}}$ is not a Schottky group. It can therefore be approximated by dense representations of $B_{i}$ (as in the proof of Lemma 3.2 of [Mi]). It follows that $\rho_{i}$ can be approximated by redundant representations of $F_{n}$. Since $\mathcal{P} S_{n}^{2}$ is open, we conclude that $\mathcal{P} S_{n}^{2} \cap\left(\overline{\mathcal{R}}_{n}(G) \times \overline{\mathcal{R}}_{n}(G)\right)$ is nonempty.

This concludes the proof in the complex case. 
6.2. The real case. A discrete faithful representation $F_{n} \rightarrow \mathrm{SL}_{2}(\mathbb{R})$ corresponds to a Fuchsian group, and if this group is a lattice with just one parabolic then the representation is automatically in $\mathcal{P} S_{n}$, by the main theorem of [Mi]. So we have to consider groups with two or more parabolics.

Let $\Sigma$ be a sphere with $k \geq 4$ punctures. Then $\pi_{1}(\Sigma)$ can be written as a free group on $n=k-1$ letters $X_{1}, \ldots, X_{n}$, representing $k-1$ of the punctures, with the last puncture represented by the product $X_{1} X_{2} \cdots X_{n}$.

Let $g_{1}$ be a cyclically reduced word in the generators $X_{2}, \ldots, X_{n}$, such that the Whitehead graph $W_{1}=\mathrm{Wh}\left(g_{1},\left\{X_{2}, \ldots, X_{n}\right\}\right)$ is connected and without cutpoints. Let $\Phi_{1} \in \operatorname{Aut}\left(F_{n}\right)$ be the automorphism defined by

$$
\begin{aligned}
X_{1} & \mapsto X_{1} g_{1}^{m}, \\
X_{2} & \mapsto X_{2} X_{1} g_{1}^{m}, \\
& \vdots \\
X_{n} & \mapsto X_{n} X_{1} g_{1}^{m} .
\end{aligned}
$$

(This can be obtained as a composition of Nielsen moves, first multiplying $X_{1}$ by the letters in $g_{1}^{m}$, and then multiplying each $X_{i}$ for $i>1$ by the image of $X_{1}$.) If $m$ is chosen sufficiently large then the $\Phi_{1}$ image of each of the $k$ punctures has Whitehead graph (with respect to all the generators) containing $W_{1}$.

Let $\rho_{0}: \pi_{1}(\Sigma) \rightarrow \mathrm{SL}_{2}(\mathbb{R})$ be a discrete faithful Fuchsian representation taking all $k$ punctures to parabolics, and let

$$
\rho_{1}=\rho_{0} \circ \Phi_{1}^{-1} .
$$

Then the parabolics of $\rho_{1}$ are in $k$ conjugacy classes, each of whom by itself has Whitehead graph containing $W_{1}$.

Now define $g_{2}, W_{2}, \Phi_{2}$ and $\rho_{2}$ the same way, but interchanging the roles of $X_{1}$ with $X_{2}$. The graph $W_{2}$ is then connected and without cutpoints when restricted to the vertices associated to $X_{1}, X_{3}, \ldots, X_{n}$.

The rest of the proof goes through in essentially the same way to show that $\left(\left[\rho_{1}\right],\left[\rho_{2}\right]\right)$ is in $\mathcal{P} S_{n}^{2}$. Namely, a conjugacy class whose axis is badly non-quasigeodesic in both representations must wrap around at least one of the parabolics in $\rho_{1}$ and at least one of the parabolics in $\rho_{2}$ as well. Hence its Whitehead graph (with respect to all $n$ generators) contains $W_{1} \cup W_{2}$. Since $W_{1}$ and $W_{2}$ intersect in at least two vertices (those associated to $X_{3}$ ), their union is connected and without cutpoints.

We see that each $\rho_{i}$ is approximated by redundant representations as before, since each contains a parabolic that is inside a free factor (in fact is itself primitive).

Remark 6.5. The non-mixing result Theorem 2.2 extends to every rank one simple Lie group. Indeed, Lemma 6.2 holds in this generality and the same proof applies. The only issue that requires some justification is the non-emptiness of $\mathcal{P} S_{n}^{2} \cap \overline{\mathcal{R}}^{2}$. However, since every noncompact simple Lie group $G$ admits a subgroup $H$ locally 
isomorphic to $\mathrm{SL}_{2}(\mathbb{R})$ such that for some point $x$ in the symmetric space $G / K$ the orbit $H \cdot x$ is isometric to the hyperbolic plane $\mathbb{H}^{2}$ (cf. [PR], Theorem 3.7), this result can be deduced easily from the $\mathrm{SL}_{2}(\mathbb{R})$ analog.

\section{Some related problems}

Let us end this paper by recalling and suggesting some old and new related problems.

7.1. The other conjecture of Lubotzky. First let us repeat Lubotzky's second conjecture $[\mathrm{Lu}]$, mentioned also in [Mi], which is still a mystery, even for $\mathrm{SL}_{2}(\mathbb{R})$ and $\mathrm{SL}_{2}(\mathbb{C})$ :

Problem 7.1. Let $n \geq 3$. Given a connected simple Lie group, is it true that almost every representation of $F_{n}$ is either redundant or primitive stable?

When $k$ is non-archimedean and $G=\mathbb{G}(k)$ is the group of $k$ rational points of some Zariski connected simple algebraic group $\mathbb{G}$, Problem 7.1 still makes sense when restricting to unbounded representations. It can be deduced from [Gl] that for $\mathrm{SL}_{2}(k)$ almost every dense representation of $F_{n}$ is redundant. Hence, the question in this case is whether almost every discrete faithful representation is primitive stable or even Schottky. For higher rank groups, e.g. for $\mathrm{SL}_{3}(k)$ it is unclear if the definition of primitive stable representations extends in a useful way.

7.2. Does density of primitives imply redundant?. It is straightforward, that if $f: F_{n} \rightarrow G$ is redundant then $f\left(P_{n}\right)$ is dense in $G$, where $P_{n} \subset F_{n}$ is the set of primitive elements. Moreover, if $G$ is discrete (e.g. finite) then the opposite is also true, i.e. if $f\left(P_{n}\right)=G$ then $f$ is redundant (consider a basis containing a primitive element that maps to $1 \in G)$.

Problem 7.2. Let $G$ be a topological group, or more specifically, a simple Lie group over a local field. Is it true that every representation $f: F_{n} \rightarrow G$ for which $f\left(P_{n}\right)$ is dense, is redundant?

It could be that the answer depends on $n$.

7.3. Extending the results of this paper to semisimple groups. In this paper we restricted ourselves to the case where the group $G$ is simple rather than semisimple. However, as remarked in 2.5 , over $\mathbb{R}$ or $\mathbb{C}$, Theorems 2.1 and 2.4 remain true, with only slight modifications in the arguments, when $G$ has more than one factor. However for nonarchimedean fields, although we expect that the theorems remain valid, some parts of our proofs do not directly apply: 
Problem 7.3. Extend the result of this paper to all semisimple groups over local fields, and more generally, to groups of the form $\prod_{i=1}^{n} \mathbb{G}_{i}\left(k_{i}\right)$ where $\mathbb{G}_{i}$ are simple algebraic groups and $k_{i}$ are local fields.

7.4. Other notions of weak mixing. For a group $G$ acting on a finite measure space $X$, weak mixing is equivalent to each of the following:

(1) the action of $G$ on $X \times X$ is ergodic,

(2) for every finite measure preserving ergodic space $Y$, the action on $X \times Y$ is ergodic,

(3) the unitary representation $L^{2}(X)$ has no finite dimensional sub-representation but the constants.

For a compact Lie group $G$, since the space $\mathcal{R}_{n}(G)=\operatorname{Hom}\left(F_{n}, G\right)$ has finite measure, the three conditions above are equivalent and, as shown in [Ge], satisfied whenever $n \geq 3$.

However, when $G$ is noncompact and $n \geq 3$, the space $\mathcal{R}_{n}(G)$ is an infinite measure space, hence the various notions of weak mixing are no longer equivalent. One may still ask whether for every ergodic probability space $Y$ the action of $\operatorname{Aut}\left(F_{n}\right)$ on $\mathcal{R}_{n}(G) \times Y$ is ergodic. In particular, one may study this question in the special case $Y=\operatorname{Hom}\left(F_{n}, H\right)$ where $H$ is a connected compact group.

7.5. The notion of spread for topological groups. Recall that a finite (or discrete) group $G$ is said to have spread $k$ if for any $k$ nontrivial elements $g_{1}, \ldots, g_{k} \in G \backslash\{1\}$ there is $h \in G \backslash\{1\}$ which generates $G$ simultaneously with each of the $g_{i}$ 's, i.e. for all $i,\left\langle h, g_{i}\right\rangle=G$. Any finite simple group has spread 2 (see [GKS], [GS]). We can extend the definition of spread to topological groups by requiring that the closure $\overline{\left\langle h, g_{i}\right\rangle}$ equals $G$ for all $i$.

Let now $G$ be a connected center-free simple Lie group. By [AV] $G$ has spread 1 (see also [Ku1], [Ku2]). Additionally, given $g \in G$, it is not hard to show that if $\langle g, h\rangle$ is dense in $G$ for some $h \in G$ then the set $\{h \in G: \overline{\langle g, h\rangle}=G\}$ contains a neighborhood of the identity minus some exponential proper subvariety. Hence if $G$ has spread 1, it has spread $k$ for any finite $k$. The same result holds with respect to Zariski topology.

Similarly, one can define the notion of random-spread as follows: Say that $G$ has random-spread $k$ if for almost every $k$ elements $g_{1}, \ldots, g_{k}$ in $G$ there is a simultaneous generating partner, i.e. $h$ such that $\left\langle h, g_{i}\right\rangle$ is dense for each $i$. One can deduce from the discussion in Section 3 that every connected semisimple Lie group has random-spread $k$ for every finite $k$.

It might be interesting however to study the notion of random-spread (as well as the true spread and other variants of it) for semisimple Lie groups over non-Archimedean local fields. 


\section{References}

[AV] H. Abels and E. B. Vinberg, Generating semisimple groups by tori. J. Algebra 328 (2011), 114-121. Zbl 1220.22005 MR 2745556

[BL] Y. Barnea and M. Larsen, Random generation in semisimple algebraic groups over local fields. J. Algebra 271 (2004), 1-10. Zbl 1049.20028 MR 2022476

[BG] E. Breuillard and T. Gelander, On dense free subgroups of Lie groups. J. Algebra 261 (2003), 448-467. Zbl 1014.22007 MR 1966638

[E] M. J. Evans, T-systems of certain finite simple groups. Math. Proc. Cambridge Philos. Soc. 113 (1993), 9-22. Zbl 0781.20021 MR 1188815

[Ge] T. Gelander, On deformations of $F_{n}$ in compact Lie groups. Israel J. Math. 167 (2008), 15-26. Zbl 1171.22007 MR 2448015

[GZ] T. Gelander and A. Żuk, Dependence of Kazhdan constants on generating subsets. Israel J. Math. 129 (2002), 93-98. Zbl 0993.22003 MR 1910934

[Gi] R. Gilman, Finite quotients of the automorphism group of a free group. Canad. $J$. Math. 29 (1977), 541-551. Zbl 0332.20010 MR 0435226

[Gl] Y. Glasner, A zero-one law for random subgroups of some totally disconnected groups. Transform. Groups 14 (2009), 787-800. Zbl 1191.22001 MR 2577198

[Go] W. M. Goldman, An ergodic action of the outer automorphism group of a free group. Geom. Funct. Anal. 17 (2007), 793-805. Zbl 1139.57002 MR 2346275

[GKS] R. M. Guralnick, W. M. Kantor, and J. Saxl, The probability of generating a classical group. Comm. Algebra 22 (1994), 1395-1402. Zbl 0820.20022 MR 1261266

[GS] R. M. Guralnick and A. Shalev, On the spread of finite simple groups. Combinatorica 23 (2003), 73-87. Zbl 1027.20004 MR 1996627

[KW] I. Kapovich and R. Weidmann, Freely indecomposable groups acting on hyperbolic spaces. Internat. J. Algebra Comput. 14 (2004), 115-171. Zbl 1094.20022 MR 2058318

[Kl] F. Klein, Neue Beiträge zur Riemann'schen Functionentheorie. Math. Ann. 21 (1883), 141-218. JFM 15.0351.01

[Ku1] M. Kuranishi, Two elements generations on semi-simple Lie groups. Kōdai Math. Sem. Rep. 1 (1949), no. 5-6, 9-10. Zbl 0045.00702 MR 0034766

[Ku2] M. Kuranishi, On everywhere dense imbedding of free groups in Lie groups. Nagoya Math. J. 2 (1951), 63-71. Zbl 0045.31003 MR 0041145

[Lu] A. Lubotzky, Dynamics and $\operatorname{Aut}\left(F_{n}\right)$ actions on group presentations and representations. In Geometry, rigidity, and group actions, Chicago Lectures in Math., University of Chicago Press, Chicago 2011, 609-643. Zbl 1266.20045 MR 2807845

[Ma] B. Maskit, On Klein's combination theorem. Trans. Amer. Math. Soc. 120 (1965), 499-509. Zbl 0138.06803 MR 0192047

[Mi] Y. N. Minsky, On dynamics of $\operatorname{Out}\left(F_{n}\right)$ on $\mathrm{PSL}_{2}(\mathbb{C})$ characters. Israel J. Math. 193 (2013), 47-70. Zbl 06176901 MR 3038545

[Pi] R. Pink, Compact subgroups of linear algebraic groups. J. Algebra 206 (1998), 438-504. Zbl 0914.20044 MR 1637068 
[PR] V. Platonov and A. Rapinchuk, Algebraic groups and number theory. Pure Appl. Math. 139, Academic Press, Boston 1994. Zbl 0841.20046 MR 1278263

[Pr] G. Prasad, Elementary proof of a theorem of Bruhat-Tits-Rousseau and of a theorem of Tits. Bull. Soc. Math. France 110 (1982), 197-202. Zbl 0492.20029 MR 667750

[R] M. S. Raghunathan, Discrete subgroups of Lie groups. Ergeb. Math. Grenzgeb. 68, Springer-Verlag, Berlin 1972. Zbl 0254.22005 MR 0507234

[Sh] Y. Shalom, Rigidity of commensurators and irreducible lattices. Invent. Math. 141 (2000), 1-54. Zbl 0978.22010 MR 1767270

[St] J. R. Stallings, Whitehead graphs on handlebodies. In Geometric group theory down under (Canberra, 1996), de Gruyter, Berlin 1999, 317-330. Zbl 1127.57300 MR 1714852

[We] R. Weidmann, The Nielsen method for groups acting on trees. Proc. London Math. Soc. (3) 85 (2002), 93-118. Zbl 1018.20020 MR 1901370

[Wh1] J. H. C. Whitehead, On certain sets of elements in a free group. Proc. London Math. Soc. (2) 41 (1936), 48-56. JFM 62.0079.04 Zbl 0013.24801 MR 1575455

[Wh2] J. H. C. Whitehead, On equivalent sets of elements in a free group. Ann. of Math. (2) 37 (1936), 782-800. JFM 62.1093.04 Zbl 0015.24804 MR 1503309

Received February 3, 2012; revised April 25, 2011

T. Gelander, Einstein Institute of Mathematics, Edmond J. Safra Campus, Givat Ram, The Hebrew University of Jerusalem, Jerusalem, 91904, Israel

E-mail: gelander@math.huji.ac.il

Y. Minsky, Yale University, Mathematics Department, PO Box 208283, New Haven, CT 06520-8283, U.S.A.

E-mail: yair.minsky@yale.edu 